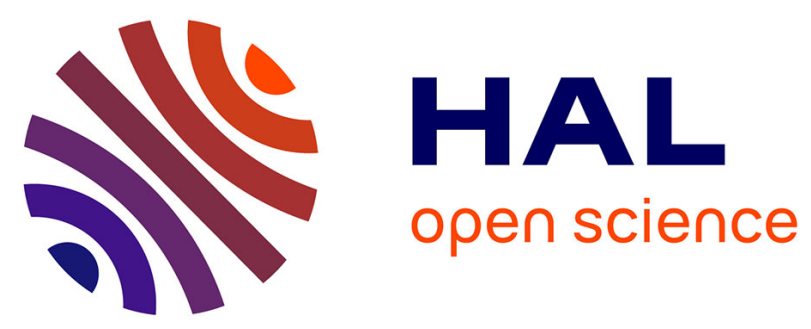

\title{
Interoperable atlases of the human brain
}

K Amunts, M.J. Hawrylycz, David C. van Essen, Jd van Horn., N Harel, Jean-Baptiste Poline, F de Martino, J. G. Bjaalie, Ghislaine Dehaene-Lambertz, Stanislas Dehaene, et al.

\section{- To cite this version:}

K Amunts, M.J. Hawrylycz, David C. van Essen, Jd van Horn., N Harel, et al.. Interoperable atlases of the human brain. NeuroImage, 2014, 99 (1), pp.8. 10.1016/j.neuroimage.2014.06.010 . hal-01094749

\section{HAL Id: hal-01094749 \\ https://inria.hal.science/hal-01094749}

Submitted on 3 Jan 2015

HAL is a multi-disciplinary open access archive for the deposit and dissemination of scientific research documents, whether they are published or not. The documents may come from teaching and research institutions in France or abroad, or from public or private research centers.
L'archive ouverte pluridisciplinaire HAL, est destinée au dépôt et à la diffusion de documents scientifiques de niveau recherche, publiés ou non, émanant des établissements d'enseignement et de recherche français ou étrangers, des laboratoires publics ou privés. 


\section{Comments and Controversies}

\section{Interoperable atlases of the human brain}

K. Amunts a,b , M.J. Hawrylycz c , D.C. Van Essen ${ }^{\text {d }}$, J.D. Van Horn ${ }^{\text {e }}$, N. Harel ${ }^{\text {f }}$, J.-B. Poline ${ }^{g}$, F. De Martino ${ }^{\text {h }}$, J.G. Bjaalie ${ }^{\mathrm{i}}$, G. Dehaene-Lambertz ${ }^{\mathrm{j}}$, S. Dehaene ${ }^{\mathrm{j}}$, P. Valdes-Sosa ${ }^{\mathrm{k}, \mathrm{l}}$, B. Thirion ${ }^{\mathrm{m}}$, K. Zilles ${ }^{\text {n,o }}$, S.L. Hill ${ }^{\mathrm{p}}$, M.B. Abrams ${ }^{\text {p,* }}$, P.A. Tass ${ }^{\mathrm{a}, \mathrm{q}, \mathrm{r}}$, W. Vanduffel ${ }^{\text {s }}$, A.C. Evans ${ }^{\mathrm{t}}$, S.B. Eickhoff ${ }^{\mathrm{a}, \mathrm{u}}$

a Institute of Neuroscience and Medicine, INM-1, Research Centre Jülich, Germany

b C. and O. Vogt Institute for Brain Research, Heinrich Heine University, Düsseldorf, Germany

${ }^{c}$ Allen Institute for Brain Science, Seattle, WA, USA

d Department of Anatomy and Neurobiology, Washington University School of Medicine, St. Louis, MO, USA

e The Institute for Neuroimaging and Informatics (INI) and Laboratory for Neuro Imaging (LONI), Keck School of Medicine, University of Southern California, Los Angeles, CA, USA

${ }^{\mathrm{f}}$ Center for Magnetic Resonance Research, Departments of Radiology \&' Neurosurgery, University of Minnesota School of Medicine, Minneapolis, MN, USA

${ }^{g}$ Hellen Wills Neuroscience Institute, Brain Imaging Center, University of California at Berkeley, CA, USA

${ }^{\mathrm{h}}$ Department of Cognitive Neuroscience, Faculty of Psychology and Neuroscience, Maastricht University, Maastricht, The Netherlands

i Institute of Basic Medical Sciences, University of Oslo, Oslo, Norway

j INSERM, U992, Cognitive Neuroimaging Unit, F-91191 Gif/Yvette, France

${ }^{\mathrm{k}}$ Cuban Neuroscience Center, Havana, Cuba

${ }^{1}$ Key Laboratory for Neuroinformation, Chengudu, China

${ }^{m}$ Parietal Research Team, French Institute for Research in Computer Science and Automation (INRIA), Gif sur Yvette, France

${ }^{n}$ Department of Psychiatry, Psychotherapy and Psychosomatics, RWTH University Aachen, Aachen, Germany

o Jülich-Aachen Research Alliance (JARA), Translational Brain Medicine, Jülich, Germany

${ }^{\mathrm{P}}$ International Neuroinformatics Coordinating Facility Secretariat (INCF), Stockholm, Sweden

${ }^{q}$ Department of Neuromodulation, University of Cologne, Cologne, Germany

${ }^{r}$ Department of Neurosurgery, Stanford University, Stanford, USA

${ }^{s}$ Department of Neurosciences, KU Leuven, Leuven, Belgium

${ }^{\mathrm{t}}$ Montreal Neurological Institute, McGill University, Montreal, Canada

u Institute for Clinical Neuroscience and Medical Psychology, Heinrich-Heine University, Düsseldorf, Germany

\section{A R T I C L E I N F O}

\section{Article history:}

Accepted 2 June 2014

Available online $\mathrm{xxxx}$

\begin{abstract}
A B S T R A C T
The last two decades have seen an unprecedented development of human brain mapping approaches at various spatial and temporal scales. Together, these have provided a large fundus of information on many different aspects of the human brain including micro- and macrostructural segregation, regional specialization of function, connectivity, and temporal dynamics. Atlases are central in order to integrate such diverse information in a topographically meaningful way. It is noteworthy, that the brain mapping field has been developed along several major lines such as structure vs. function, postmortem vs. in vivo, individual features of the brain vs. population-based aspects, or slow vs. fast dynamics. In order to understand human brain organization, however, it seems inevitable that these different lines are integrated and combined into a multimodal human brain model. To this aim, we held a workshop to determine the constraints of a multi-modal human brain model that are needed to enable (i) an integration of different spatial and temporal scales and data modalities into a common reference system, and (ii) efficient data exchange and analysis. As detailed in this report, to arrive at fully interoperable atlases of the human brain will still require much work at the frontiers of data acquisition, analysis, and representation. Among them, the latter may provide the most challenging task, in particular when it comes to representing features of vastly different scales of space, time and abstraction. The potential benefits of such endeavor, however, clearly outweigh the problems, as only such kind of multi-modal human brain atlas may provide a starting point from which the complex relationships between structure, function, and connectivity may be explored.
\end{abstract}

(c) 2014 Elsevier Inc. All rights reserved.

\section{Introduction}

The last two decades have seen remarkable advances in human brain mapping at multiple spatial and temporal scales. Together, these

\footnotetext{
* Corresponding author at: Nobels vägen 15A SE-171 77 Stockholm, Sweden

E-mail address: mathew.abrams@incf.org (M.B. Abrams).
}

developments have provided a large corpus of information about many different aspects of human brain organization, including microand macro-structure, regional specialization of function, (structural and functional) connectivity, as well as temporal dynamics. They have also led to specialized brain mapping subfields along several major lines, such as structure vs. function, postmortem vs. in vivo, individual features vs. population-based aspects, as well as slow vs. fast dynamics 
(Toga et al., 2006). In order to relate these different aspects to each other and understand the organization of the human brain, it is necessary to achieve closer integration across modalities. One key aspect of this involves multi-modal human brain atlases. The concept of a multi-modal atlas is not new - different modalities ranging from cytoarchitecture or gene expression data to activity and connectivity maps identified through functional imaging have been mapped in the past, and combined into a common reference space (e.g., Eickhoff et al., 2005; Hawrylycz et al., 2012; Toga et al., 2006; Van Essen et al., 2012). A frequently used volumetric reference space is the Montreal Neurological Institute (MNI) space for which thousands of individual data sets have been collected in the past (Evans et al., 1992, 2012). Different modalities can thus be compared using the topography represented by MNI space as a framework.

This approach, while having proven invaluable for reconciling distinct data sets into a common 3-dimensional coordinate framework, also has drawbacks. In order to take full advantage of a multi-modal approach, a comprehensive brain atlas must go beyond a simple superimposition of individual datasets or aspects of brain organization and instead should integrate data across multiple modalities as accurately as possible as a prerequisite for quantitative analyses of their interrelationships. Meeting this objective entails compensating for individual variability in functional and microstructural organization rather than just using shape features (e.g., folding patterns) for inter-subject alignment. In addition, several constraints must be respected to enable the integration of different spatial and temporal scales, as well as different data modalities into a common reference system for efficient data exchange, visualization, and analysis.

In June of 2013, the International Neuroinformatics Coordinating Facility (INCF) held a workshop as a satellite event of OHBM 2013 in Seattle, WA, USA, entitled Towards a multi-modal human brain atlas, which brought together scientists from the different brain mapping fields who aim to identify the limitations of such multi-modal human brain model and to identify potential solutions to these constraints. The participants agreed that given the highly diverse needs of different fields within neuroscience with respect to standards and templates, a single template or reference brain for all applications should be inadequate. Instead, the multi-modal human brain model is envisioned to reside in multiple, interoperable reference spaces. It is also clear, however, that an open-ended number of reference spaces and templates poses a challenge because unconstrained proliferation would negate the very idea of a reference space. Moreover, there is only one reference space presently available, the BigBrain (Amunts et al., 2013), which is capable of integrating data about the microstructure of the human brain, or results of physiological recordings of small networks while considering the topography of the brain at a spatial resolution of $20 \mu \mathrm{m}$. Thus, the community needs to define the rules for how to navigate among different spaces, to develop an approach that considers how a multi-modal brain atlas is built, and to set the criteria for quality of certain reference spaces or templates. Here, we propose some steps in that direction and present a summary of the key challenges impeding this vision of interoperable multi-modal human brain atlases as well as a potential roadmap towards such models.

Before starting, we would like to point out, that while presenting challenges and potential solutions to these that are generic to brain atlas development, we focus our presentation and the provided examples on mapping the gray matter of the human brain based on structure, function and connectivity. Conversely, the construction of white matter atlases based (primarily) on diffusion-weighted imaging (e.g., Durrleman et al., 2011; Oishi et al., 2008; Prasad et al., 2014; Thiebaut de Schotten et al., 2011; Zhang et al., 2010), their integration with those representing gray matter features or cross-species comparisons (Dougherty et al., 2005; Jbabdi et al., 2013; Javad et al., 2014; Sallet et al., 2013; Thiebaut de Schotten et al., 2012; Yendiki et al., 2011) will not be in the focus of the present work. We hope that this constraint will allow us to provide a more coherent overview on the state of the field, the challenges towards a true multi-modal brain atlas and potential solutions to overcome these.

\section{Key challenges for a multi-modal human brain atlas}

Interoperability between reference spaces, templates, and other atlases

The spatial superposition and comparison of different templates containing different sets of data will be crucial for accurate integration of different sources of knowledge and multi-modal mapping of the human brain. As a result, establishing precise and representationally valid mappings between different standard spaces and templates is of utmost importance towards the development of a multi-modal human brain atlas (Zilles and Amunts., 2010), especially in light of the ever-increasing number of sub-group specific templates (i.e. for different age groups, ethnicities, and pathologies, cf. Fonov et al., 2011).

In this text, template refers to an exemplary brain scan or an aggregate of brain scans, which are possibly multi-modal and often averaged across multiple subjects. A space in this context is simply the coordinate system associated with a specific template. We can think of an atlas as a way to label this (or these) image(s), deciding where the structures or features described by the atlas lie using the template coordinate system (cf. Fig. 1). In short, an atlas can be defined as a mapping between a template and a probability distribution associated with the set of labels. The mapping can simply be from one point in the coordinate system $(x, y, z)$ to one specific label, but the definition above would account for probabilistic atlases. Each position may have different labels: For example, one and the same region in a brain can anatomically be labeled based on its location in a particular gyrus, lobe, Brodmann area, cytoarchitectonic area represented as a probabilistic map, cortical layer, area defined by gene expression patterns or connectivity, functional response pattern, etc.

While the methods for brain image registration are constantly improving (Klein et al., 2009, 2010), mapping between templates or between individual subjects and a particular template is severely impaired by what has been termed the correspondence problem; biologically, it has its origin in the inter-individual variability in size, shape, and morphology of human brains. For example, not all macroscopic brain landmarks (sulci, gyri, etc.) are present in the same way in each subject, especially for the highly convoluted cerebral cortex (Ono et al., 1990).

Inter-subject variability and methodically induced variability interact and may influence biological parameters represented in a template brain. In particular, the following factors are relevant:

I. normal variability depends upon the brain region - areas of Broca's region are more variable, for example, than the primary visual cortex and the striatum

II. normal variability depends upon the spatial scale investigated the occipital lobe is always at the back of the brain, but its shape, sulcal pattern (secondary and tertiary sulci in particular), and the location of individual cortical areas are variable (Caspers et al., 2013)

III. the global features of the brain introduce some variability, a brain of an Alzheimer patient will have different parameters than that of a young adult or a newborn

IV. the method employed for spatial normalization, e.g. the degree of freedom for achieving spatial correspondence and the amount of subsequent spatial smoothing

V. reproducibility of the respective feature, i.e., the amount of noise that is associated with its observation

There are many cases where a one-to-one mapping of morphological features between (template) brains is not feasible, e.g. when a 


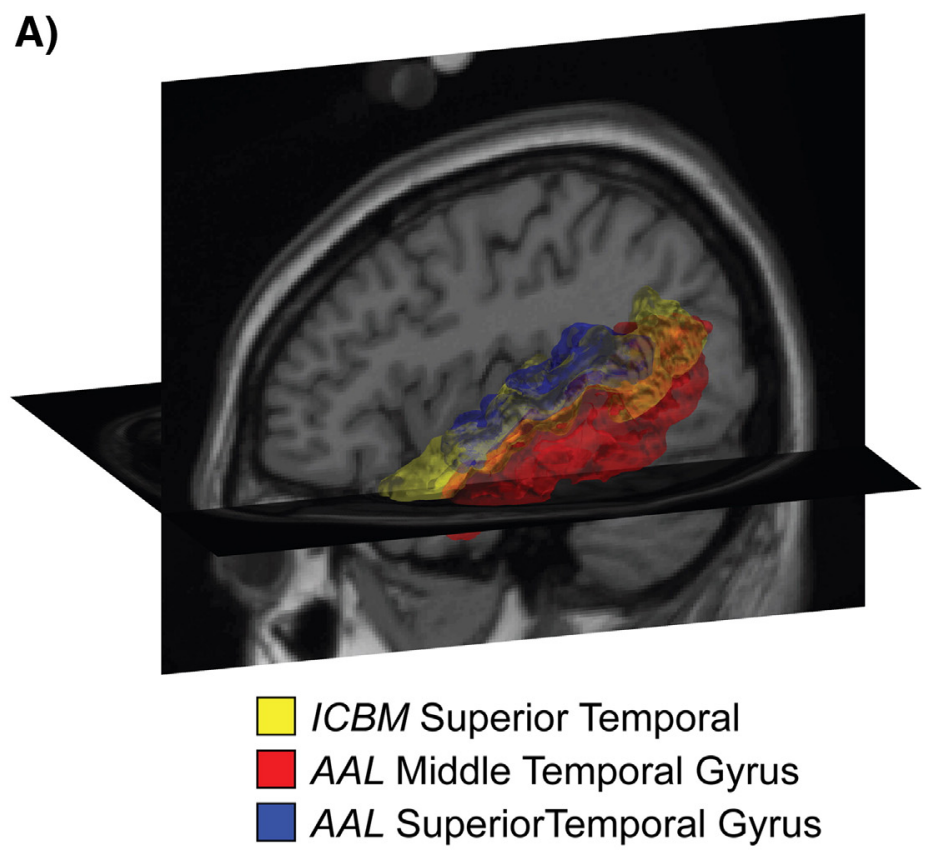

B)
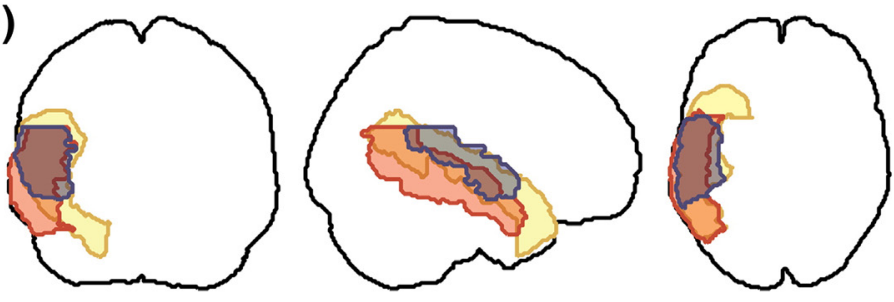

C)
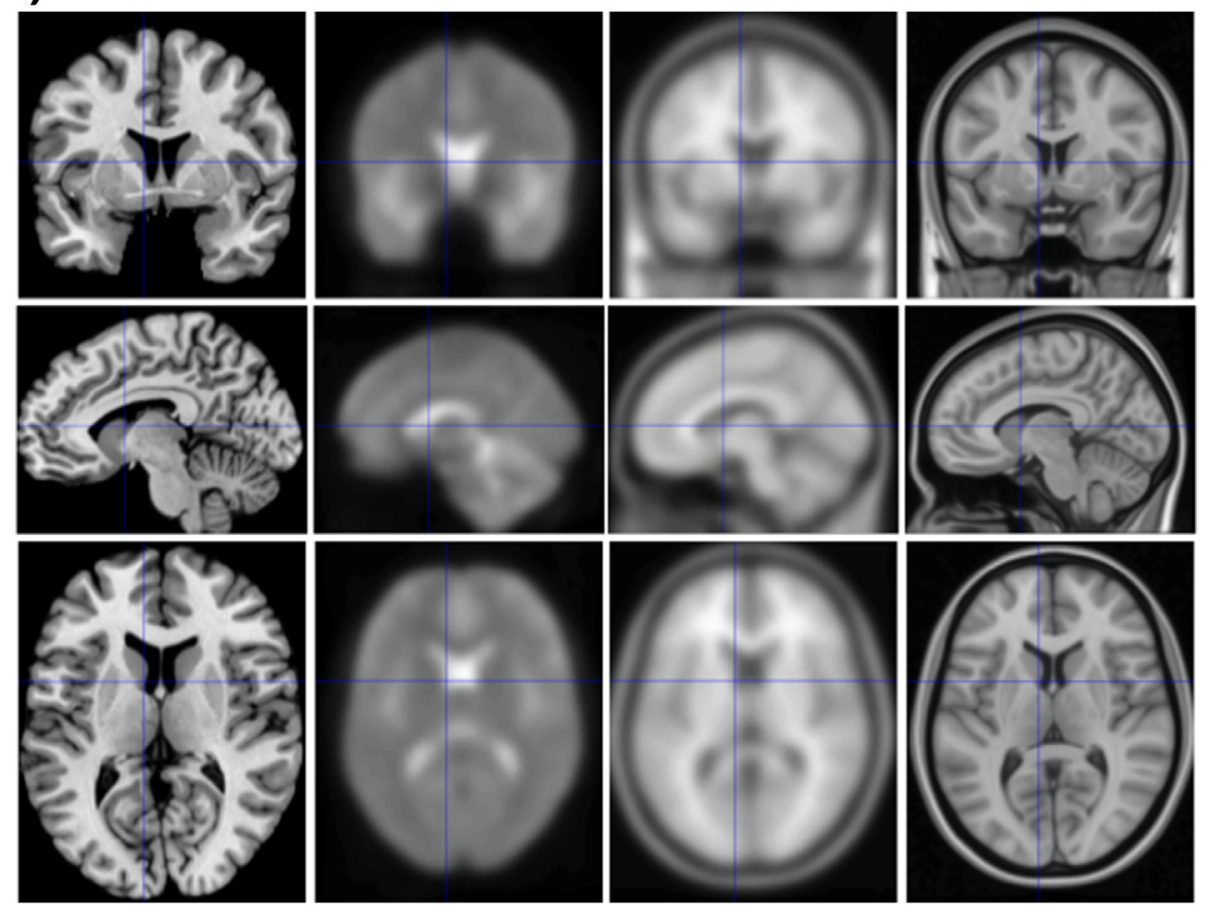

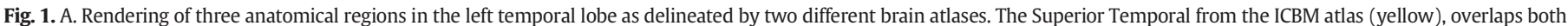

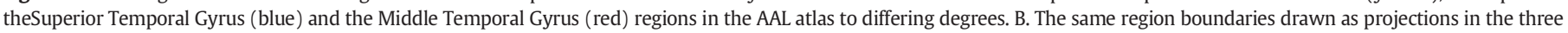

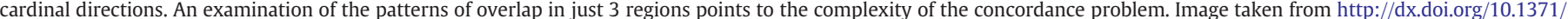

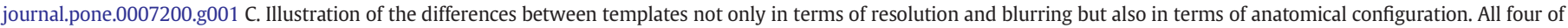

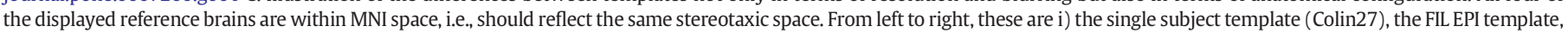
the MNI152 linear average and the MNI152 non-linear average template. 
particular sulcus is duplicated or branched in one brain, but not in another. In addition, mapping gross anatomical landmarks on each other usually implies that there is a correspondence between the various local structural and functional features, an assumption that is often not proven. Consequently, aligning gross morphology, until recently the standard approach for matching between subjects and templates, may not always represent the optimal way to map between labels (Brett et al., 2002; Tucholka et al., 2012; Robinson et al., in press; Smith et al., 2013).

Furthermore, the relationship between brain morphology and the representations of cortical areas or functional specializations is also variable (Amunts et al., 2004; Eickhoff et al., 2009). Hence, enforcing registration purely based on shape characteristics may lead to suboptimal alignment of functional neuroanatomy, increased variance and a loss of biological validity. Methods based on functional alignment have become more and more applied - they approach the problem "from the other site", which makes sense for solving many scientific questions (Dumoulin and Wandell, 2008; Frost and Goebel, 2012; cf. Javad et al., 2014). Both approaches are relevant. Evidence has been provided last time, that the relationship between sulcal morphology and functional parcellation is close in regions beyond primary cortical areas, e.g., the fusiform gyrus (Weiner et al., 2014). Since other areas such as areas 44 and 45 of Broca's region seem to exhibit a less strong relationship, it would be relevant to address the variable relationship between both aspects of brain organization in a systematic way. However, the degree to which idiosyncrasies and variability should be handled in the spatial mapping between individuals, templates, and the ensuing atlases remains a challenge (Devlin and Poldrack, 2007) for which multi-modal registration algorithms are certainly part of the solution (Sabuncu et al., 2010).

It should be noted that even when a one-to-one mapping is likely to exist, the community has not always agreed on some common procedure to identify regions or landmark. A striking example of the current situation has been presented in Bohland et al., 2009. This work demonstrates that even undisputed brain regions (e.g., the superior temporal gyrus) may have little correspondence between different atlases. Efforts to specify a standard procedure for labeling regions and/or landmarks in the normal population are therefore critical to our ability to refer to the same location across subjects (Klein and Tourville, 2012), and need to be integrated in the relevant software packages. The variability induced by the spatial registration procedures adds to current mismatch of labels between studies.

\section{Heterogeneity and variability of features}

Features can be classified according to certain aspects of brain organization, e.g., structure, function, and (functional or anatomical) connectivity (Eickhoff and Grefkes, 2011). Within these axes, features can be differentiated, which represent distinct, though not always independent, information. For example, the density of cell bodies ("structure") is inversely correlated with the neuropil, i.e., space occupied by synapses, dendrites, and axons ("connectivity"). In addition, the attribution to one of these axes may not be unambiguous; e.g., the expression of a certain transcription factor or a receptor for a certain neurotransmitter can be interpreted both in terms of structure or function.

When comparing one and the same feature between different brains, inter-subject differences occur. Limiting these differences just to "noise", which results in a "signal loss", would not be adequate. Rather inter-individual differences (inter-subject variability) is an important topic of research as it may contain important information, for example for a certain cognitive experience or ability (Schlaug et al., 1995). In fact, relating inter-individual differences in brain structure to behavioral phenotype may represent a most powerful approach to inferring functional correlates of inter-personally variable anatomy (e.g., Durrleman et al., 2011; Thiebaut de Schotten et al., 2011). Following the notion that inter-subject variability may be a key component of understanding brain organization, most current brain atlases are probabilistic by their very nature (e.g., Forkert et al., 2012; Mazziotta et al., 2001; Roland and Zilles, 1994; Sun et al., 2012) and hence reflect this important aspect of brain organization.

Another important though often neglected scale is that of intersubject averaging - within the analysis of a neuroimaging experiment and also in the creation of that template itself (e.g., the MNI 305, MNI 152, etc.; Evans et al., 2012). Many spatial features of brain organization show marked inter-individual differences and become lost when averaging across subjects. The orientation columns in the visual cortex provide the best example of such a feature. While these may be clearly identified in individual subjects, their arrangement and number is highly variable across subjects, resulting in a loss of feature information when pooling over different individuals (Yacoub et al., 2008).

At the other end of the averaging spectrum are those features that can only be identified at the group level such as task-based coactivation patterns that emerge from the aggregation of hundreds of different neuroimaging experiments collectively probing a vast multitude of paradigms (Eickhoff et al., 2010) or structural covariance maps that are calculated by the correlation of anatomical features across subjects (Evans, 2013). Mapping the human brain and creating a probabilistic multi-modal atlas will provide information that allows inference on features and their variability across a population, as illustrated by multimodal data obtained from the Human Connectome Project (Smith et al., 2013). The relationship between features that i) may bridge across scales, those that ii) are only describable at the individual level, and those that iii) are defined by across subject relationships are important frontiers for investigation.

Finally it has to be mentioned that the quality and meaning of features also depends on methodological aspects. The spatial scale of many features depends on the amount of deformation that is enforced during the spatial normalization, e.g. the parameterization and regulation of the registration method, necessary to switch from one representation to the other and/or filtering applied prior to data analysis. That is, there can be a complex interaction between the spatial scale of features that can be represented in a map, spatial registration, the choice of the template and of the employed atlas. As a simple example, it has repeatedly been shown that functional maps may be markedly different depending on whether analysis is carried out on volume- or surfacebased templates following volume- and surface-based registration, respectively (Tucholka et al., 2012; Van Essen et al., 2012).

\section{Time-dependency of atlas information}

Although mapping the human brain and creating a multi-modal atlas is intrinsically a spatial endeavor, the fact that features may be expressed at very different time scales should not be ignored. On the upper end of these temporal scales, changes during evolution (Mantini et al., 2012; Orban et al., 2004; Sallet et al., 2013) and over lifespan, both in development and aging, entail a massive effect on any attempt to characterize brain structure, function, and connectivity (Dougherty et al., 2005). Consequently, any map of regional organization must be considered a reflection of a particular developmental stage. This issue is further complicated by the fact that lifetime trajectories may differ over subjects, rendering brain maps substantially more variable during periods of intensive development (e.g. during infancy and childhood as well as aging) or in the presence of pathological processes. Although such differences in trajectories and in regional interindividual variability are well acknowledged in the respective research fields, attempts to capture them into a spatio-temporal framework are few.

At the other temporal extreme, oscillatory brain activity and synchronization of neuronal networks in the range of milliseconds represent the finest temporal scale that may be resolved by today's human neuroimaging methods. Given their dynamic and often contextdependent nature as well as their much coarser spatial resolution 
(e.g., in EEG or MEG data), such information is obviously difficult to present in an atlas based on spatial templates. As a result, little effort has yet been made to integrate information on the topographic distribution of features on fast temporal scales into human brain atlases. Nevertheless, dynamic features like electrical transient and oscillations do have spatial properties as evident when looking at generator analyses or power maps and there may thus be the potential to include features that live on a faster temporal scale into a multi-modal brain atlas.

\section{Integration of information from different subjects and experiments}

As stated above, an atlas can be defined as a mapping from one or more (individual or group) template, which define the spatial framework, to a feature or label. This is different from traditional postmortem atlases, e.g., Brodmann's cytoarchitectonic map (1909), which is based on a single brain and single modality - cytoarchitecture. New atlas systems such as the JuBrain atlas have been constructed by registering multiple brains on a template in order to represent inter-individual correspondence in a probabilistic way and allow the integration across modalities (Eickhoff et al., 2005; Zilles and Amunts, 2010). As a consequence, the association of any other data to this atlas can only be probabilistic. That is, different within one and the same system of labels, features that may be mutually exclusive in an individual (two different histological areas) may be associated with different probabilities to one and the same position.

Atlas labels represent particular spatially constrained properties of a specific cortical or subcortical location, e.g., a cytoarchitectonic area, a functional response, a connection pattern, or the location on a given gyrus. Numerical representation of labels or brain signals and their probability/intensity are often called maps. The labeling of any given location may be either probabilistic (in which each location is assigned a probability for each of the different labels in the respective map) or deterministic (in which the mapping attributes to each location one and only one label, i.e., one label has probability one whereas the probability for all other labels is zero). Probabilistic labels are particularly common in population-based mapping where they denote, e.g. the percentage of subjects featuring a particular characteristic at any given location. In turn, deterministic atlases may be derived from parcellations in a single brain (e.g. by labeling anatomical features) or as the result of a hard parcellation from probabilistic atlases (e.g., by computing maximum probability maps).

In that context, it may be noted that some atlases may not cover all brain regions, i.e., may not represent a complete map. In other words, some location in space may have a probability of zero for each label. Examples are atlases for specific structure such as the thalamus and basal ganglia Morel and Duvernoy's atlas of the brain stem and cerebellum (Morel et al., 1997; Naidich et al., 2009). Atlases that are yet under development, i.e., for which not all labels are yet available, such as the JuBrain or the Brainnetome atlases, provide a related but in some aspects also distinct case. In particular, a probability of zero for all labels in an atlas covering a specific structure indicates that the current voxel is outside that structure. In a yet incomplete atlas, it could also be found at a not yet mapped region.

One important endeavor in the context of atlas generation is to integrate the wealth of individual maps and data (in particular those on task-based functional neuroimaging data) into a larger framework of a database. Coordinate databases like BrainMap, and Neurosynth, storing the location of significant effects in many individual experiments, represent important first steps into this direction as they, together with a robust taxonomy of experimental designs, allow meta-scale integration on neuroimaging data and quantitative functional decoding (Laird et al., 2009, 2011).

Integrating the intrinsically heterogeneous and noisy information provided by the current neuroimaging literature with maps derived from other modalities, such as anatomical features or connectivity, remains an important challenge for the generation of a multi-modal atlas (see Fig. 2). This can be facilitated by improvements in data acquisition and analysis (cf. Smith et al., 2013; Van Essen et al., 2013).

\section{Labeling regions or mapping features?}

Several questions regarding the ultimate goal of human brain atlasing arise from the inherent spatial complexity of the human brain. The brain may be parcellated (by deterministic labels or maximum probability maps) or (probabilistically) labeled by a large number of regionally specific properties: Is then the goal to identify and delineate distinct regions in the brain, i.e., regions that are maximally different from each other and maximally homogeneous within them? Or rather is the goal to provide a multivariate and probabilistic description for each voxel of one or several template spaces? Note that the second goal is more ambitious and one should relatively more easily be able to achieve the first than the second. One of the particular challenges in the latter is that atlases may not align as discussed above (atlas correspondence problem), which will make it difficult for any modality to inform another.

A detailed multivariate description of each brain location (voxel or surface vertex) in an atlas has the seeming advantage in that more flexible assumptions are made with respect to the underlying functional organization. In turn, such atlases have substantial drawbacks when it comes to labeling a particular location - the problem of defining where "I am" is merely postponed to later steps of analysis. Conversely, approaches aimed at parcellating the brain into distinct regions provide a counter advantage, as they allow for an easy communication of where in the brain a particular property is located, albeit at the expense of not reflecting the heterogeneous nature of regional differentiation and providing a "static" representation dependent on the set of features and the specific algorithm used for labeling.

Last, note that there is a fundamental difference between a brain characteristic that has a true probabilistic nature (e.g. the amount or percentage of free dopaminergic receptors in a given regions) and the "pseudo probabilistic" nature because frequencies are computed (e.g., the hand motor cortex is located anterior to the central sulcus, but averaging hand movement fMRI dataset will show some probability that it is posterior to the sulcus because of the registration problem).

\section{What do we need to build a better multi-modal brain atlas?}

The challenges for the construction of a conceptual and computational infrastructure supporting reliable and scientifically meaningful information on human brain organization are great - as are the expected benefits. Clearly this infrastructure has to be dynamic, integrating semantic technologies and including the notion of versions and provenance, and therefore is likely to rely on modern web based software development. To be successful, this construction will also have to be tied to efforts in the development of brain structure and function ontologies, access to data with neuroimaging data-sharing initiatives (Poline et al., 2012) and databasing (Laird et al., 2011), closely linked to the developments and advances in registration and machine learning methods for landmarks identification, and to advance in data acquisitions and modeling. Establishing the standards in the human brain atlasing domain is crucial, however, for our scientific community and will not succeed if not undertaken by an international effort.

Based on the topics discussed above, we think that the following aspects have to be considered to advance atlasing the human brain (cf. schematic summary in Fig. 3):

Establish reliable and anatomically precise mappings between different templates and atlas spaces

There are highly diverse needs of different fields within neuroimaging with respect to standards and templates, and a single reference brain may not fit applications. In order to integrate information on the 


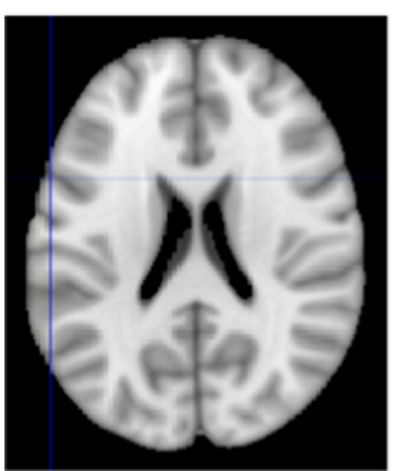

MNI coordinates: -59 / 11 / 21

Left frontal cortex

Harvard-Oxford Cortical Atlas (probabilistic macroanatomy) $32 \%$ Precentral Gyrus,

$32 \%$ Inferior Frontal Gyrus, pars opercularis

AAL (deterministic macroanatomy)

Inferior frontal gyrus, part opercularis

JuBrain (probabilistic microanatomy)

$65 \%$ cytoarchitectonic Area 44

$35 \%$ cytoarchitectonic Area 44
Structural connectivity (tractography)
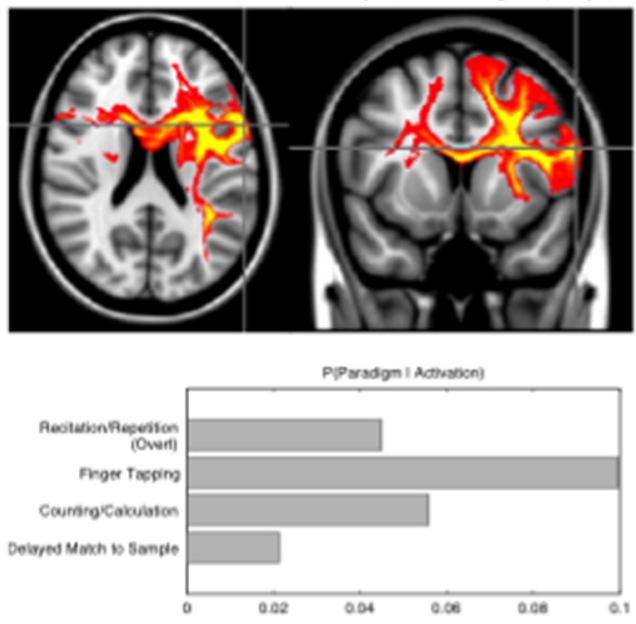

Quantitative reverse inference
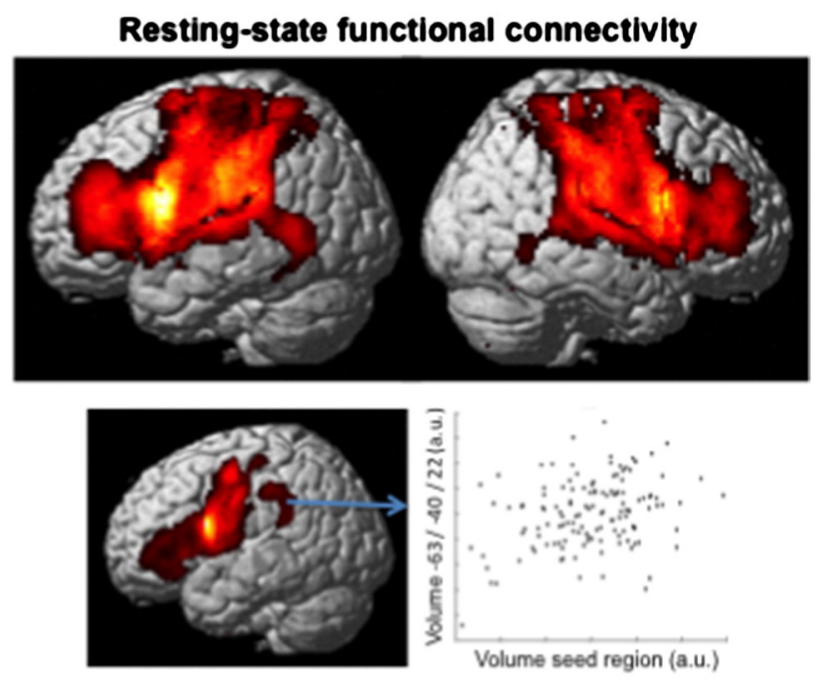

Structural covariance (130 subjects)

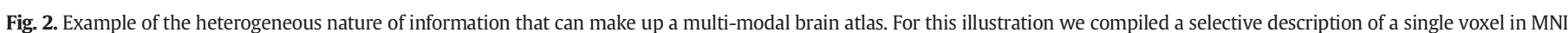

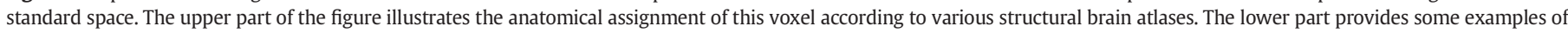

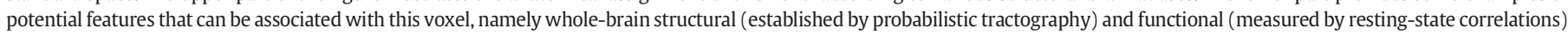
connectivity patterns, quantitative reverse inference by means of the BrainMap database and structural covariance measurements in a cohort of healthy subjects.

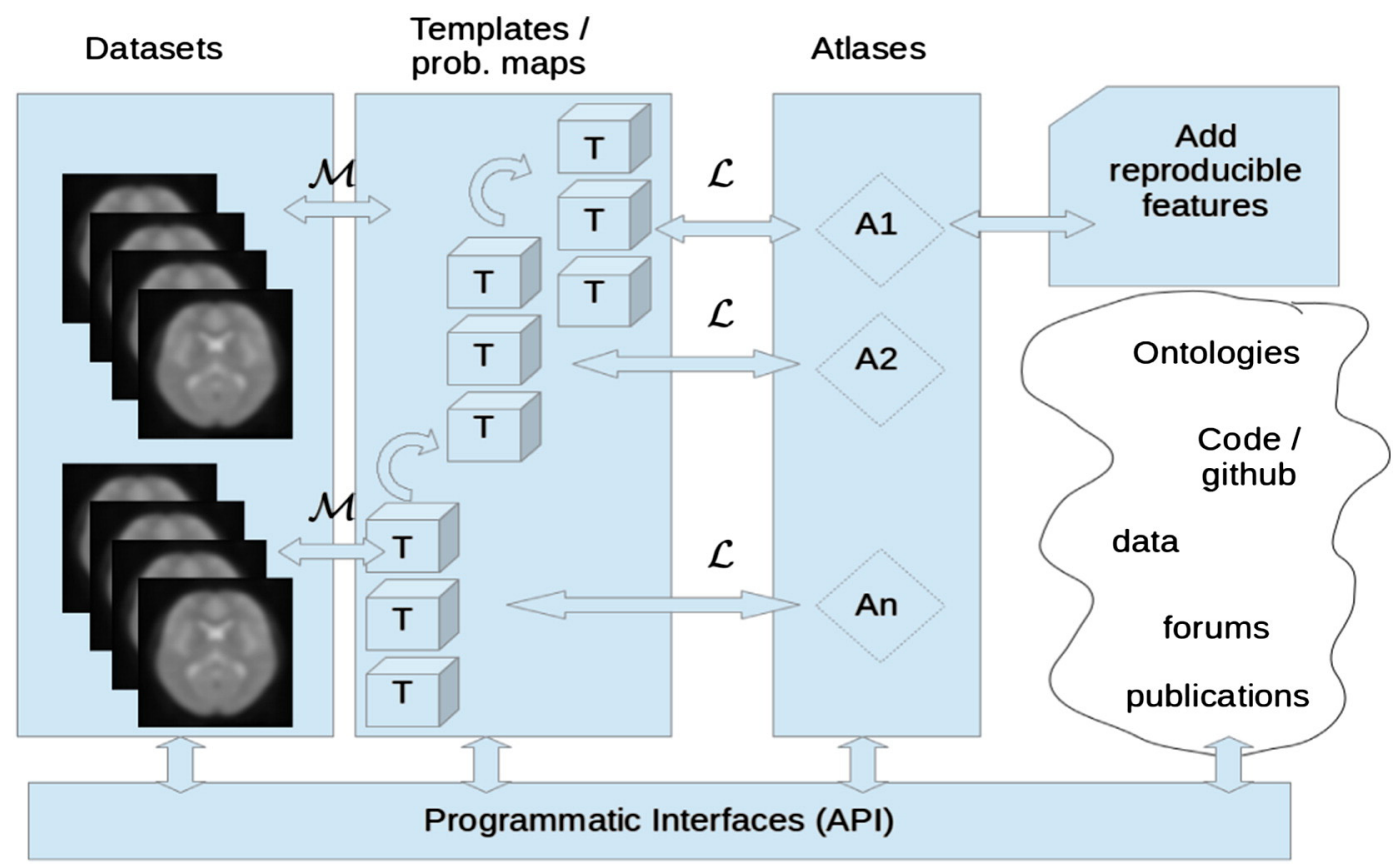

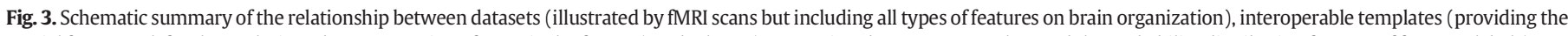

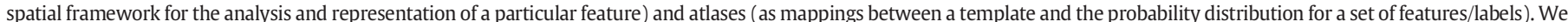

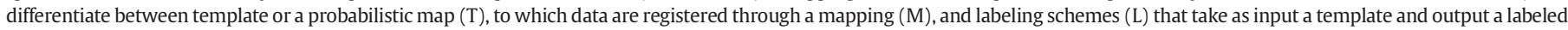

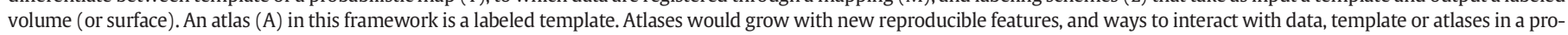
grammatic way would benefit from open-science projects in the neuroinformatic domain and web based discussions within the brain imaging community. 
most diverse aspects of brain organization into a truly multi-modal brain atlas, this atlas may thus live in various template and associated spaces bridged by established pipelines that make them interoperable. To define the procedures to interoperate between them, to quantify the error of transformation between them, and to estimate the quality of a certain atlas or reference space are tasks that have yet to be resolved in order to handle the various spaces and templates. For the cerebral cortex, it is particularly important to optimize the alignment of functional regions rather than the underlying pattern of gyri and sulci. Fortunately, recently reported methods for function-based intersubject alignment hold great promise for improving the fidelity of human cortical alignment (Conroy et al., 2013; Robinson et al., in press; Smith et al., 2013).

Provide a framework that may integrate heterogeneous information at different levels of abstraction

As noted, some features such as orientation sensitivity or oscillatory behavior may only be fully understood at time-, space-, or averagingscales, which may have a complex or even unknown relationship to a reference space. Other important features such as individual task fMRI results reflect information that in isolation may only be of limited use, and may not allow generalization. To integrate the diverse nature of features into a (spatial) brain atlas may thus require compromises with respect to the amount of (temporal or context-dependent) information that may be retained. Finding approaches that either abstract their spatial properties or provide large-scale agglomeration across individual findings will thus be an important step towards the integration of these aspects into a multi-modal atlas.

\section{Distinguish between labels and descriptions}

A multi-modal brain atlas will need a coordinate system with a (limited) set of robust labels denoting distinct regions attached to these coordinates that map between the different templates. It is necessary to identify robust parcellations, i.e., parcellations, which can be reproduced in (almost) all brains, as these labels will facilitate communication between features and investigators. Cytoarchitecture provides a strong basis in that respect (Zilles and Amunts, 2010), as it represents a basic architectonic framework of cortical organization. To which extent such label-framework based on static, structural aspects is suited to label dynamic, functional properties though remains to be explored. By the integration of a large amount of features reflecting regional properties in structure, function, and connectivity, however, the atlas will provide a detailed multivariate description for each voxel of the interoperable template spaces.

\section{Provide a spatial framework that accommodates multi-scalar data}

A multi-modal atlas that has some longevity in brain mapping research must acknowledge that data will be collected at increasingly fine spatial scales. At present, atlases that are used for neuroimaging experiments are defined on a $1 \mathrm{~mm}$ 3D grid. As imaging technology advances and as we seek to incorporate high-resolution $(<10 \mu \mathrm{m})$ data from invasive or post-mortem techniques, we need a spatial framework that bridges these scales. The recent BigBrain dataset (Amunts et al., 2013) is one example of a higher $(20 \mu \mathrm{m})$ resolution 3D cytoarchitectonic map that would be a template for integrating imaging data and gene expression data from the Allen Brain atlas (Hawrylycz et al., 2012). In this context, we would like to note that it seems inevitable for such high-resolution templates to be based on individual subjects, as inter-subject averaging defies the whole purpose of representation at the micrometer level. This again highlights the essential nature of establishing precise mappings between different template spaces for interoperability.
In summary, we conclude that to arrive at fully interoperable atlases of the human brain will still require much work at the frontiers of data acquisition, analysis and representation. Among them, the latest may provide the most challenging tasks, in particular when it comes to representing features of vastly different scales of space, time and abstraction. The potential benefits of such endeavor, however, clearly outweigh the problems, as only such kind of multi-modal human brain atlas may provide a starting point from which the complex relationships between structure, function and connectivity may be explored.

\section{Acknowledgment}

This work is the outcome of a workshop sponsored by the International Neuroinformatics Coordinating Facility (INCF) on digital brain atlasing. INCF is an international organization launched in 2005, following a proposal from the Global Science Forum of the OECD to establish international coordination and collaborative informatics infrastructures for neuroscience - and currently has 17 member countries across North America, Europe, Australia, and Asia. INCF establishes and operates scientific programs to develop standards for neuroscience data sharing, analysis, modeling and simulation while coordinating an informatic infrastructure designed to enable the integration of neuroscience data and knowledge worldwide and catalyze insights into brain function in health and disease.

\section{References}

Amunts, K., Lepage, C., Borgeat, L., Mohblerg, H., Dickscheid, T., Rousseau, M.-E., Bludau, S., Bazin, P.-L., Lewis, L.B., Oros-Peusquena, A.-M., Shah, N.J., Lippert, T., Zilles, K., Evans, A.C., 2013. The Big Brain - an ultra-high resolution 3D human brain model. Science 340 (6139), 1472-1475.

Amunts, K., Weiss, P.H., Mohlberg, H., Pieperhoff, P., Eickhoff, S., Gurd, J., Shah, J.N., Marshall, J.C., Fink, G.R., Zilles, K., 2004. Analysis of the neural mechanisms underlying verbal fluency in cytoarchitectonically defined stereotactic space - the role of Brodmann's areas 44 and 45. Neuroimage 22 (1), 42-56.

Bohland, J.W., Bokil, H., Mitra, P.D., 2009. The brain atlas concordance problem: quantitative comparison of anatomical parcellations. PLoS One 4 (9), E7200.

Brett, M., Johnsrude, I.S., Owen, A.M., 2002. The problem of functional localization in the human brain. Nat. Rev. Neurosci. 3 (3), 243-249.

Caspers, J., Zilles, K., Eickhoff, S.B., Schleicher, A., Mohlberg, H., Amunts, K., 2013. Cytoarchitectonical analysis and probabilistic mapping of two extrastriate areas of the human posterior fusiform gyrus. Brain Struct. Funct. 218, 511-526.

Conroy, B., Singer, B., Guntupalli, J., Ramadge, P., Haxby, J., 2013. Intersubject alignment of human cortical anatomy using functional connectivity. Neuroimage 81, 400-411.

Devlin, J.T., Poldrack, R.A., 2007. In praise of tedious anatomy. Neuroimage 37 (4), 1033-1041 (discussion 1050-8).

Dougherty, R.F., Ben-Shachar, M., Deutsch, G., Potanina, P., Bammer, R., Wandell, B.A., 2005. Occipital-callosal pathways in children: validation and atlas development. Ann. N. Y. Acad. Sci. 1064, 98-112.

Dumoulin, S.O., Wandell, B.A., 2008. Population receptive field estimates in human visual cortex. Neuroimage 39 (2), 647-660.

Durrleman, S., Fillard, P., Pennec, X., Trouvé, A., Ayache, N., 2011. Registration, atlas estimation and variability analysis of white matter fiber bundles modeled as currents. Neuroimage 55 (3), 1073-1090.

Eickhoff, S.B., Grefkes, C., 2011. Approaches for the integrated analysis of structure, function and connectivity of the human brain. Clin. EEG Neurosci. 42, 107-121.

Eickhoff, S.B., Jbabdi, S., Caspers, S., Laird, A.R., Fox, P.T., Zilles, K., Behrens, T.E., 2010. Anatomical and functional connectivity of cytoarchitectonic areas within the human parietal operculum. J. Neurosci. 30, 6409-6421.

Eickhoff, S.B., Laird, A.R., Grefkes, C., Wang, L.E., Zilles, K., Fox, P.T., 2009. Coordinate-based activation likelihood estimation meta-analysis of neuroimaging data: a randomeffects approach based on empirical estimates of spatial uncertainty. Hum. Brain Mapp. 30, 2907-2926.

Eickhoff, S.B., Stephan, K.E., Mohlberg, H., Grefkes, C., Fink, G.R., Amunts, K., Zilles, K., 2005. A new SPM toolbox for combining probabilistic cytoarchitectonic maps and functional imaging data. Neuroimage 25, 1325-1335.

Evans, A.C., Janke, A.L., Collins, D.L., Baillet, S., 2012. Brain templates and atlases. Neuroimage 62, 911-922.

Evans, A.C., Marrett, S., Neelin, P., Collins, L., Worsley, K., Dai, W., Milot, S., Meyer, E., Bub, D., 1992. Anatomical mapping of functional activation in stereotactic coordinate space. Neuroimage 1, 43-53.

Evans, A.C., 2013. Networks of anatomical covariance. Neuroimage 80, 489-504.

Fonov, V., Evans, A.C., Botteron, K., Almli, C.R., McKinstry, R.C., Collins, D.L., 2011. Unbiased average age-appropriate atlases for pediatric studies. Neuroimage 54, 313-327.

Forkert, N.D., Suniaga, S., Fiehler, J., Wersching, H., Knecht, S., Kemmling, A., 2012. Generation of a probabilistic arterial cerebrovascular atlas derived from 700 time-of-flight MRA datasets. Stud. Health Technol. Inform. 180, 148-152. 
Frost, M.A., Goebel, R., 2012. Measuring structural-functional correspondence: spatial variability of specialised brain regions after macroanatomical alignment. Neuroimage 59 (2), 1369-1381

Hawrylycz, M.J., Lein, E.S., Guillozet-Bongaarts, A.L., Shen, E.H., Ng, L., Miller, J.A., van de Lagemaat, L.N., Smith, K.A., Ebbert, A., Riley, Z.L., Abajian, C., Beckmann, C.F., Bernard, A., Bertagnolli, D., Boe, A.F., Cartagena, P.M., Chakravarty, M.M., Chapin, M., Chong, J., Dalley, R.A., Daly, B.D., Dang, C., Datta, S., Dee, N., Dolbeare, T.A., Faber, V., Feng, D., Fowler, D.R., Goldy, J., Gregor, B.W., Haradon, Z., Haynor, D.R., Hohmann, J.G., Horvath, S., Howard, R.E., Jeromin, A., Jochim, J.M., Kinnunen, M., Lau, C., Lazarz, E.T. Lee, C., Lemon, T.A., Li, L., Li, Y., Morris, J.A., Overly, C.C., Parker, P.D., Parry, S.E., Reding, M., Royall, J.J., Schulkin, J., Sequeira, P.A., Slaughterbeck, C.R., Smith, S.C., Sodt, A.J., Sunkin, S.M., Swanson, B.E., Vawter, M.P., Williams, D.E., Wohnoutka, P., Zielke, H.R., Geschwind, D.H., Hof, P.R., Smith, S.M., Koch, C., Grant, S.G.N., Jones, A.R., 2012. An anatomically comprehensive atlas of the adult human brain transcriptome. Nature 489 , 391-399.

Javad, F., Warren, J.D., Micallef, C., Thornton, J.S., Golay, X., Yousry, T., Mancini, L., 2014. Auditory tracts identified with combined fMRI and diffusion tractography. Neuroimage 84, 562-574.

Jbabdi, S., Lehman, J.F., Haber, S.N., Behrens, T.E., 2013. Human and monkey ventral prefrontal fibers use the same organizational principles to reach their targets: tracing versus tractography. J. Neurosci. 33 (7), 3190-3201.

Klein, A., Andersson, J., Ardekani, B.A., Ashburner, J., Avants, B., Chiang, M.C., Christensen, G.E., Collins, D.L., Gee, J., Hellier, P., Song, J.H., Jenkinson, M., Lepage, C., Rueckert, D., Thompson, P., Vercauteren, T., Woods, R.P., Mann, J.J., Parsey, R.V., 2009. Evaluation of 14 nonlinear deformation algorithms applied to human brain MRI registration. Neuroimage 46 (3), 786-802

Klein, A., Ghosh, S.S., Avants, B., Yeo, B.T., Fischl, B., Ardekani, B., Gee, J.C., Mann, J.J., Parsey, R.V., 2010. Evaluation of volume-based and surface-based brain image registration methods. Neuroimage 51 (1), 214-220.

Klein, A., Tourville, J., 2012. 101 labeled brain images and a consistent human cortical labeling protocol. Front. Neurosci. 6, 171.

Laird, A.R., Eickhoff, S.B., Fox, P.M., Uecker, A.M., Ray, K.L., Saenz, J.J., McKay, D.R., Bzdok, D., Laird, R.W., Robinson, J.L., Turner, J.A., Turkeltaub, P.E., Lancaster, J.L., Fox, P.T., 2011. The BrainMap strategy for standardization, sharing, and meta-analysis of neuroimaging data. BMC Res. Notes 4, 349.

Laird, A.R., Eickhoff, S.B., Rottschy, C., Bzdok, D., Ray, K.L., Fox, P.T., 2009. Networks of task co-activations. Neuroimage 80, 505-514.

Mantini, D., Hasson, U., Betti, V., Perrucci, M., Romani, G., Corbetta, M., Orban, G., Vanduffel, W., 2012. Interspecies activity correlations reveal functional correspondence between monkey and human brain areas. Nat. Methods 9 (3), 277-282.

Mazziotta, J.A., Toga, A.W., Evans, A.C., Fox, P.T., Lancaster, J., Zilles, K., Woods, R., Paus, T., Simpson, G., Pike, B., Holmes, C., Collins, D.L., Thompson, P., MacDonald, D., Iacoboni, M., Schormann, T., Amunts, K., Palomero-Gallagher, N., Geyer, S., Parsons, L., Narr, K. Kabani, N., LeGoualher, G., Boomsma, D., Cannon, T., Kawashima, R., Mazoyer, B. 2001. A probabilistic atlas and reference system for the human brain: International Consortium for Brain Mapping (ICBM). Philos. Trans. R. Soc. Lond. B Biol. Sci. 356, 1293-1322.

Morel, A., Magnin, M., Jeanmonod, D., 1997. Multiachitectonic and stereotactic atlas of the human thalamus. J. Comp. Neurol. 387, 588-630.

Naidich, T.P., Duvernoy, H.M., Delman, B.N., Sorensen, A.G., Kollias, S.S., Haacke, E.M. 2009. Duvernoy's Atlas of the Human Brain Stem and Cerebellum. Springer, Wien.

Oishi, K., Zilles, K., Amunts, K., Faria, A., Jiang, H., Li, X., Akhter, K., Hua, K., Woods, R., Toga, A.W., Pike, G.B., Rosa-Neto, P., Evans, A., Zhang, J., Huang, H., Miller, M.I., van Zijl, P.C., Mazziotta, J., Mori, S., 2008. Human brain white matter atlas: identification and assignment of common anatomical structures in superficial white matter. Neuroimage 43 (3), 447-457.

Ono, M., Kubik, S., Abernathey, C.D., 1990. Atlas of Cerebral Sulci. Thieme, Stuttgart, (Print).

Orban, G., Van Essen, D., Vanduffel, W., 2004. Comparative mapping of higher visual areas in monkeys and humans. Trends Cogn. Sci. 8 (7), 315-324.

Poline, J.-B., Breeze, J.L., Ghosh, S.S., Gorgolewski, K., Halchenko, Y.O., Hanke, M., Helmer, K.G., Marcus, D.S., Poldrack, R.A., Schwartz, Y., Ashburner, J., Kennedy, D.N., 2012. Data sharing in neuroimaging research. Front. Neuroinform. 6, 1-13.
Prasad, G., Joshi, S.H., Jahanshad, N., Villalon-Reina, J., Aganj, I., Lenglet, C., Sapiro, G. McMahon, K.L., de Zubicaray, G.I., Martin, N.G., Wright, M.J., Toga, A.W., Thompson, P.M., 2014. Automatic clustering and population analysis of white matter tracts using maximum density paths. Neuroimage 15 (97), 284-295.

Robinson, E.C., Jbabdi, S., Glasser, M.F., Andersson, J., Burgess, G.C., Harms, M.P., Smith, S.M., Van Essen, D.C., Jenkinson, M., 2014. MSM: a new flexible framework for multimodal surface matching. Neuroimage. http://dx.doi.org/10.1016/j.neuroimage.2014.05.069 (in press).

Roland, P.E., Zilles, K., 1994. Brain atlases-a new research tool. Trends Neurosci. 17 (11), 458-467.

Sabuncu, M.R., Singer, B.D., Conroy, B., Bryan, R.E., Ramadge, P.J., Haxby, J.V., 2010. Function-based inter-subject alignment of the cortical anatomy. Cereb. Cortex 20, $130-140$.

Sallet, J., Mars, R.B., Noonan, M.P., Neubert, F.X., Jbabdi, S., O'Reilly, J.X., Filippini, N., Thomas, A.G., Rushworth, M.F., 2013. The organization of dorsal frontal cortex in humans and macaques. J. Neurosci. 33 (30), 12255-12274.

Schlaug, G., Jäncke, L., Huang, Y., Steinmetz, H., 1995. In vivo evidence of structural brain asymmetry in musicians. Science 267, 699-701.

Smith, S.M., Vidaurre, D., Beckmann, C.F., Glasser, M.F., Jenkinson, M., Miller, K.L., Nichols, T.E., Robinson, E., Salimi-Khorshidi, G., Woolrich, M.W., Barch, D.M., Ugurbil, K., Van Essen, D.C., 2013. Functional connectomics from resting-state fMRI. Trends Cogn. Sci. 80, 144-168.

Sun, Z.Y., Klöppel, S., Rivière, D., Perrot, M., Frackowiak, R., Siebner, H., Mangin, J.F., 2012 The effect of handedness on the shape of the central sulcus. Neuroimage 60 (1) 332-339.

Toga, A.W., Thompson, P.M., Mori, S., Amunts, K., Zilles, K., 2006. Towards multimodal atlases of the human brain. Nat. Rev. Neurosci. 7, 952-966.

Thiebaut de Schotten, M., Ffytche, D.H., Bizzi, A., Dell'Acqua, F., Allin, M., Walshe, M. Murray, R., Williams, S.C., Murphy, D.G., Catani, M., 2011. Atlasing location, asymmetry and inter-subject variability of white matter tracts in the human brain with MR diffusion tractography. Neuroimage 54 (1), 49-59.

Thiebaut de Schotten, M., Dell'Acqua, F., Valabregue, R., Catani, M., 2012. Monkey to human comparative anatomy of the frontal lobe association tracts. Cortex 48 (1), 82-96.

Tucholka, A., Fritsch, V., Poline, J.-B., Thirion, B., 2012. An empirical comparison of surfacebased and volume-based group studies in neuroimaging. Neuroimage 63, 1443-1453.

Van Essen, D.C., Smith, S.M., Barch, D.M., Behrens, T.E.J., Yacoub, E., Ugurbil, K., for the WUMinn HCP Consortium, 2013. The WU-Minn Human Connectome Project: an overview. Neuroimage 80, 62-79.

Van Essen, D.C., Glasser, M.F., Dierker, D., Harwell, J., Coalson, T., 2012. Parcellations and hemispheric asymmetries of human cerebral cortex analyzed on surface-based atlases. Cereb. Cortex 22, 2241-2262.

Weiner, K.S., Golarai, G., Caspers, J., Chuapoco, M.R., Mohlberg, H., Zilles, K., Amunts, K. Grill-Spector, K., 2014. The mid-fusiform sulcus: a landmark identifying both cytoarchitectonic and functional divisions of human ventral temporal cortex. Neuroimage 84, 453-465.

Yacoub, E., Harel, N., Uğurbil, K., 2008. In vivo architectonics: a cortico-centric perspective. Proc. Natl. Acad. Sci. U. S. A. 105 (30), 10607-10612.

Yendiki, A., Panneck, P., Srinivasan, P., Stevens, A., Zöllei, L., Augustinack, J. Wang, R., Salat, D., Ehrlich, S., Behrens, T., Jbabdi, S., Gollub, R., Fischl, B., 2011. Automated probabilistic reconstruction of white-matter pathways in health and disease using an atlas of the underlying anatomy. Front. Neuroinform. 5, 23.

Zhang, Y., Zhang, J., Oishi, K., Faria, A.V., Jiang, H., Li, X., Akhter, K., Rosa-Neto, P., Pike, G.B Evans, A., Toga, A.W., Woods, R., Mazziotta, J.C., Miller, M.I., van Zijl, P.C., Mori, S., 2010. Atlas-guided tract reconstruction for automated and comprehensive examination of the white matter anatomy. Neuroimage 52 (4), 1289-1301.

Zilles, K., Amunts, K., 2010. Centenary of Brodmann's map-conception and fate. Nat. Rev. Neurosci. 11, 139-145. 